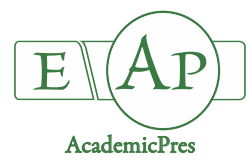

\title{
Transcriptome Sequencing of Two Kentucky Bluegrass (Poa pratensis L.) Genotypes in Response to Heat Stress
}

\author{
Qiong $\mathrm{LI}^{1}$, Yali $\mathrm{HE}^{2}$, Mingyue $\mathrm{TU}^{1}$, Junhui $\mathrm{YAN}^{1}$, Liangliang $\mathrm{YU}^{1}$, \\ Weiwei $\mathrm{QI}^{1}$, Xiaojun YUAN ${ }^{1 *}$ \\ ${ }^{1}$ Shanghai University, School of Life Science, Shanghai Key Laboratory of Bio-Energy Crops, Shanghai 200444, \\ China; liqiongsh@126.com;m18817510336@163.com;junhuiyan@shu.edu.cn;yuliangliang@shu.edu.cn;weiweiqi@shu.edu.cn; \\ yuanxiaojun@shu.edu.cn (*correspondingauthor) \\ 2Shanghai Jiaotong University, School of Agriculture and Biology, Shanghai 200240,China; heyali@sjtu.edu.cn
}

\begin{abstract}
Kentucky bluegrass (Poa pratensis L.) (KBG) is a major cool-season turfgrass. However, as its complex genetic background and production modes, limited genomic and transcriptomic information of KBG was known so far. In this study, a transcriptome study between wild type material Ninglan (summer stress sensitive) and cultivar material KBG03 (summer stress tolerant) was conducted, under optimal $\left(25^{\circ} \mathrm{C}\right)$ and high $\left(40{ }^{\circ} \mathrm{C}\right)$ temperatures. A total of $81.42 \mathrm{~Gb}$ clean reads were generated and de novo assembled into 110,784 unigenes with an average length of $1,105 \mathrm{bp}$. About 50\% KBG unigenes were similar to the non-redundant (NR) database. Among the NR BLASTx top hits, $27.47 \%$ unigenes were matched to Brachypodium distachyon. After heat stress, a massive amount of unigenes showed significantly differential expression in both genotypes. After $2 \mathrm{~h}$ heat stress, more specially up-regulated differentially expressed unigenes (DEGs) and less down-regulated DEGs were detected in Ninglan than in KBG03. At 24h, the expression of 1710 and 730 unigenes were increased and decreased uniquely in Ninglan, and 1361 up-regulated DEGs and 757 down-regulated DEGs were just found in KBG03. More heat shock proteins (HSPs) and heat shock transcription factors (HSFs) DEGs were also identified at $2 \mathrm{~h}$ than $24 \mathrm{~h}$ in both genotypes. In addition, by Gene Ontology (GO) enrichment analysis, three core terms ("protein folding", "response to heat", and "response to hydrogen peroxide") of biological process (BP) ontology were found in both genotypes under different heat stress time. The DEGs shared in both genotypes might be related to the basic mechanism of thermal response in KBG.
\end{abstract}

Keywords: heat stress; transcriptome sequencing; turfgrass; unigenes

\section{Introduction}

Kentucky bluegrass (Poa pratensis L.) (KBG) is a perennial cool-season turfgrass that is extensively distributed in temperate regions of the world. Due to its softness, medium- to fine-leaf texture, high shoot density, good spring green-up rate and outstanding recuperative capacity (Huff, 2003), it is widely used on golf courses, athletic fields, road sides and home lawns (Puyang et al., 2015). Within $\mathrm{KBG}$, chromosome numbers range from 24 to 124 , and the base chromosome number is 7. A complex series of polyploidy and aneuploidy often exists among different strains (Huff, 2003). Their seeds could be produced by both asexually (apomixis) and sexually. Furthermore, the levels of apomixis among different genotypes vary considerably (Meyer, 1982).
As global warming intensifies, the threat on plants by high temperature has become more and more serious. It is considered as heat stress that temperature rises beyond a threshold $\left(10-15{ }^{\circ} \mathrm{C}\right.$ above ambient) and it may cause irreversible damage to plant growth and development (Wahid et al., 2007). The extent to heat damage on plants is a complex issue, depending on many factors (intensity, duration and rate of increase in temperature, when and where the high temperature occurs etc.) (Sung et al., 2003; Weis and Berry, 1988). As a complex trait, heat tolerance of plants is controlled by multiple genes. Comparing with warm-season grass, high temperature is more detrimental to cool-season grass. Heat injuries would eventually lead to growth inhibition, ion flux reduction, toxic compounds and reactive oxygen species (ROS) accumulation (Howarth, 2005). 
As an effective research method, RNA sequencing (RNA-seq) was widely applied in turfgrass and forage grass transcriptomic studies. By Illumina high-throughput sequencing, three Brachypodium sylvaticum populations from Spain, Greece and Corvallis, USA were characterized (Fox et al., 2013). The first transcriptome analysis of two buffalograss (Bouteloua dactyloides) cultivars (Wachholtz et al., 2013) and four different guinea grass (Panicum maximum Jacq.) genotypes (Toledo-Silva et al., 2013) were also performed. De novo transcriptome assemblies from multiple tissues of two perennial ryegrass (Lolium perenne L.) genotypes (Farrell et al., 2014), four Lolium-Festuca complex species (Czaban et al., 2015), two Hemarthria materials (Huang et al., 2016) were subsequently reported. Furthermore, many de novo transcriptome analyses were also used to identify the differentially expressed genes (DEGs) responsible for certain specific trait within contrary types of materials. In switchgrass (Panicum virgatum L.), the buds transcriptome of two types of tillering mutants (hightillering and low-tillering) was analyzed (Xu et al., 2015). The mining of genes relating to anthocyanin biosynthesis associated with tissue-specific pigmentation was performed in two zoysiagrass (Zoysia japonica Steud.) cultivars with purple and green spikes and stolons, respectively (Ahn et al., 2015). Dinkins et al. (2017) constructed a tall fescue (Lolium arundinaceum) transcriptome and compared gene expression profiles for endophyte-symbiotic vs. endophytefree clones in different tissues. In KBG, two dwarf mutants were analyzed for the DEGs compared with the wild type by the comprehensive transcriptomic analysis (Gan et al., 2016), and the genes related to cuticular wax deposition between leaf non-elongation zone and the emerged blade zone were also identified (Ni et al., 2016).

In the field of research on biotic and abiotic stress, more and more applications of whole RNA-seq were reported in turfgrass. For instance, perennial ryegrass transcriptome changes between resistance and susceptible genotypes with three different treatments were analyzed during early infection by snow mould (Microdochium nivale) (Kovi et al., 2016). In order to identify defense-related genes, Amaradasa and Amundsen (2016) compared transcriptome of two leaf spot susceptible and two resistant buffalograss lines with inoculated and uninoculated of Curvularia inaequalis. de novo assembly of Sclerotinia homoeocarpa L. and creeping bentgrass (Agrostis stolonifera L.) transcriptomes were done by 454 sequencing technology. Several DEGs were identified from either the host or the pathogen during the infection (Orshinsky et al., 2012). By de novo sequencing assembly of barnyardgrass (Echinochloa crus-galli), potential herbicide resistance genes from susceptible and resistant biotypes with and without herbicides treatment were identified (Yang et al., 2013). The similar research on herbicide (paraquat) resistance was performed in goosegrass (Eleusine indica L.) (An et al., 2014). A KBG transcriptome study between salt tolerant and susceptible accessions was conducted under control and salt treatments (Bushman et al., 2016). In order to improve our current understanding of the cold/freezing response and obtain the potential resistance genes, comparisons of transcriptome profiles under different temperature treatments were conducted in sheepgrass (Leymus chinensis) (Chen et al., 2013), KBG (Zhang et al., 2016) and zoysiagrass (Zoysia spp. Willd.) (Wei et al., 2015). By comparing the gene expression profiles of two tall fescue (Festuca arundinacea Schreb.) genotypes (heat tolerant and heat sensitive), certain candidate genes response to heat stress were revealed ( $\mathrm{Hu}$ et al., 2014).

Although Illumina sequencing technology was widely used to detect the DEGs related to heat stress in various plants, but it was limited applied in turfgrass. Besides RNAseq, suppression subtractive hybridization (SSH) and gene chips were adopted to analyze the difference of transcriptome. For example, between two fescue (Festuca sp.) genotypes with different heat tolerance, the transcripts of the heat tolerance-related genes were identified by SSH (Zhang et al., 2005). Using gene chips, the DEGs in response to long-term heat stress treatment were analyzed in switchgrass, and some core genes were selected to identify the heat sensitive plants ( $\mathrm{Li}$ et al., 2013). Using the Brachypodium genome array, Priest et al. (2014) presented an overall differential expression analysis of Brachypodium transcriptome after chilling, high-salinity, drought, and heat stress.

As a non-model species, we know little genomic and transcriptomic information about KBG, so RNA-seq is a valuable tool for studying the response genes under heat stress. Several transcripome sequencing researches on KBG were reported in recent years, which were focus on the cold stress, salt stress, dwarf and wax deposition (Gan et al., 2016; Ni et al., 2016; Bushman et al., 2016; Zhang et al., 2016). This is the first report of transcriptome profiling of KBG in response to heat stress. In the present study, we analyzed the transcriptome of two KBG genotypes under normal and heat stress conditions using RNA-seq. The objective of this study was to analyze the differences between diverse genotypes on a transcriptome scale, and to reveal the DEGs responded to heat stress. It could provide us some useful information to understand the genes and genetic pathways relating to heat response in KBG.

\section{Materials and Methods}

\section{Plant materials and treatments}

Two KBG materials (KBG03 and Ninglan) were used in the study. KBG03 is a new breeding line, with the characteristics of narrow leaves and light green color, which was bred by School of Agriculture and Biology, Shanghai Jiaotong University, Shanghai, China. As a wild type material, Ninglan was collected from Ningxia Province of China, which was provided by Ningxia University. Contrasting to KBG03, Ninglan exhibits deep green and wide leaves. Through field tests for several years, KBG03 appeared obvious summer stress tolerance in Shanghai and surrounding areas in summer, but Ninglan did not (data not shown). The two genotype materials were preserved and reproduced by tillering in the field.

Eight individual plants (clones) of each material were transplanted from field to plastic pots $\left(10 \times 10 \times 10 \mathrm{~cm}^{3}\right)$ filled with horticultural medium [peat:vermiculite $=7: 3$ 
330

$(\mathrm{v}: \mathrm{v})]$, and maintained in greenhouse under natural conditions for about two weeks. Then these clones were transferred to illumination incubator (HGZ-300, Shanghai Huitai Equipment manufacturing Co., Ltd.) with constant $25^{\circ} \mathrm{C}$ and full light (about $400 \mu \mathrm{mol} \mathrm{m} \mathrm{s}^{-1}$ ), and irrigated every other day. After one week, the temperature was increased rapidly to $40{ }^{\circ} \mathrm{C}$ (heat stress temperature) in 15 minutes. Equal leaf samples of each pot were harvested, and they were mixed by genotypes. In all, six sequencing samples were obtained from the two genotypes at $0 \mathrm{~h}, 2 \mathrm{~h}$ and $24 \mathrm{~h}$ heat stress treatment points, counted as 03-0h (KBG03 at 0h), 03-2h (KBG03 at 2h), 03-24h (KBG03 at 24h), NL$0 \mathrm{~h}$ (NingLan at $0 \mathrm{~h}$ ), NL-2h (NingLan at $2 \mathrm{~h}$ ) and NL-24h (NingLan at 24h). These samples were immediately frozen in liquid nitrogen for RNA extraction. Two untreated samples (03-0h and NL-0h) were used as controls.

\section{$R N A$ isolation and $R N A$ sequencing}

The total RNA of each sample was isolated using the RNeasy Plant Mini Kit (Qiagen, USA) according to the manufacturer's instructions. The quality of each RNA samples was evaluated using electrophoresis in 1\% agarose gels, and quantified by a Nanodrop 2000 machine (Thermo Scientific, Delaware, USA). The six cDNA libraries' preparation and Illumina $\mathrm{HiSeq}^{\mathrm{TM}} 2500$ paired-end sequencing were conducted by OE Biotech Co. Ltd. (Shanghai, China). The raw sequence data are available at NCBI under Bio-project number PRJNA383017 and SRA accession SRP104019.

\section{Sequence assembly and annotation}

The raw cDNA reads from RNA-Seq were filtered to obtain clean reads for data analysis. During pretreatment trimming, the raw reads with ambiguous 'N' nucleotides and low-quality sequences (quality threshold of 20, length threshold of $35 \mathrm{bp}$ ) were discarded. Then the clean reads from all libraries were further de novo assembled using Trinity software (version: trinityrnaseq_r20131110) (Grabherr et al., 2011). The assembled unigenes were aligned against public databases, including NCBI nonredundant (NR), SwissProt, Gene Ontology (GO), clusters of orthologous groups for eukaryotic complete genomes (KOG), and Kyoto Encyclopedia of Genes and Genomes (KEGG), with a significance threshold of e $<1 \mathrm{e}-5$.

\section{Identification of differentially expressed unigenes}

We calculated the expression levels of the unigenes by reads per kilobase of exon model per million mapped reads (FPKM) method (Trapnell et al., 2010). Hierarchical clustering analysis for common unigenes from the six samples was constructed using the software Cluster 3.0. A fold-change $>2$ and false discovery rate $(\mathrm{FDR})<0.05$ were used as standards to determine the significant differences in unigene expression between any two samples. DEGs were subjected to GO enrichment analysis, using the hypergeometric distribution test.

Validation of $R N A-S e q$ by Quantitative real time PCR (qRT-PCR)

Ten DEGs were chosen for validation using qRT-PCR. Total RNA (1ug) of each sample was reverse-transcribed to cDNA using SuperScript II reverse transcriptase (Invitrogen, China). Primers (Table S1) were designed using NCBI Primer-BLAST Tool (https://blast.ncbi.nlm.nih.gov/Blast.cgi) and synthesized by Sangon Biological Engineering Technology and Service Co. Ltd, Shanghai. The $P$. pratensis $\beta$-tubulin gene was used as an internal reference gene (Albertini et al., 2004). qRTPCR was performed on the CFX96 Real-Time PCR Detection System (Bio-Rad) using the SYBR Premix Ex TaqII (Takara, China). The thermal cycling conditions were as follows: $95^{\circ} \mathrm{C}$ for $3 \mathrm{~min}$, followed by 40 cycles of 95 ${ }^{\circ} \mathrm{C} 15 \mathrm{~s}, 58^{\circ} \mathrm{C} 30$ s, and $72{ }^{\circ} \mathrm{C} 20$ s. Relative expression levels of the unigenes were calculated by the $2^{-\Delta \Delta \mathrm{Ct}}$ method. All amplifications were performed with three replicates and the data were analyzed using Bio-Rad software.

\section{Results and Discussion}

\section{Sequencing and assembling}

A total of $675,628,156$ raw reads $(82.47 \mathrm{~Gb})$ were produced from six samples. After quality assessment and data filtering, $667,118,738$ clean reads $(81.42 \mathrm{~Gb})$ were selected and de novo assembled into 110,784 unigenes ( $\geq$ $300 \mathrm{bp}$ ) for further analysis (Table 1). Among them, 72,720 unigenes (66\%) were $\geq 500$ bp and 39433 (36\%) were $\geq$ $1000 \mathrm{bp}$. The N50 of these unigenes was $1694 \mathrm{bp}$, the average length $1105.18 \mathrm{bp}$, and the maximum length was 17,946 bp. Meanwhile, we individually assembled the data according to the genotypes. Then we obtained 333,570,076 and 333,548,662 clean reads from KBG03 and Ninglan, respectively. In KBG03, 127,500 unigenes ( $\geq 300 \mathrm{bp}$ ) were assembled, with an average length of $889.15 \mathrm{bp}$ and an N50 of $1302 \mathrm{bp}$ (Table 1). In Ninglan, 162,678 unigenes $(\geq 300$ bp) were assembled, with an average length of 815.55 bp and an N50 of $1101 \mathrm{bp}$. Evidently more reads were assembled, less and longer unigenes were obtained. In addition, the result showed more number and total length of unigenes was detected in Ninglan than KBG03.

Table 1. Summary of the unigenes of KBG species and two genotypes

\begin{tabular}{ccccccccc}
\hline & $\begin{array}{c}\text { All }>=300 \\
(\mathrm{bp})\end{array}$ & $\begin{array}{c}>=500 \\
(\mathrm{bp})\end{array}$ & $\begin{array}{c}>=1000 \\
(\mathrm{bp})\end{array}$ & $\begin{array}{c}\text { N50 } \\
(\mathrm{bp})\end{array}$ & $\begin{array}{c}\text { Total Length } \\
(\mathrm{bp})\end{array}$ & $\begin{array}{c}\text { Max Length } \\
(\mathrm{bp})\end{array}$ & $\begin{array}{c}\text { Min Length } \\
(\mathrm{bp})\end{array}$ & $\begin{array}{c}\text { Average } \\
\text { Length (bp) }\end{array}$ \\
\hline KBG & 110784 & 72720 & 39433 & 1694 & $1.22 \mathrm{E}+08$ & 17946 & 301 & 1105.18 \\
KBG03 & 127500 & 68779 & 32434 & 1302 & $1.13 \mathrm{E}+08$ & 17934 & 301 & 889.15 \\
Ninglan & 162678 & 82302 & 36179 & 1101 & $1.33 \mathrm{E}+08$ & 16679 & 301 & 815.55 \\
\hline
\end{tabular}


Functional annotation and classification of assembled unigenes

The assembled unigenes from all samples were queried against five public databases (Table 2). More than half unigenes (55,781 unigenes, $50.35 \%)$ were similar to proteins in the NR database. A total of 35,425 unigenes (31.98\%), 30,108 unigenes (27.18) and 29,210 unigenes (26.37\%) were similar to SwissProt, KOG, and GO databases, respectively. Only $8.31 \%$ of the total unigenes were annotated in KEGG database.

By BLASTx, a homology search was conducted against the NR database. The closest species was Brachypodium distachyon, with 15,322 unigenes (27.47\%) matched (Fig. 1). Followed by Hordeum vulgare subsp. vulgare and Aegilops tauschii, which showed 10,119 unigenes (18.14\%) and 9,891 unigenes (17.73\%) were similar with KBG. The other unigenes were similar to Triticum urartu (9.43\%), Oryza sativa Japonica Group (6.75\%), Triticum aestivum (4.79\%), Zea mays (3\%), Sorghum bicolor (2.51\%) and Setaria italic (2.37\%). The result was similar to other RNASeq researches in KBG (Gan et al., 2016; Bushman et al., 2016). They pointed out the species which closely related to KBG was Brachypodium, barley and other Triticeae species.

Furthermore, we functionally categorize the GO annotated unigenes, which were enriched in $64 \mathrm{GO}$ terms (at level 2) of three major functional categories (Fig. 2). One unigene may be matched to multiple GO terms. In the molecular function (MF), "binding" (GO: 0005488, $58.75 \%)$ and "catalytic activity" (GO: 0003824, 55.37\%) enriched the most unigenes. In the cellular component (CC), "cell" (GO: 0005623, 70.88\%) and "cell part" (GO: 0044464, 70.66\%) enriched the largest percentage of unigenes, followed by "organelle" (GO: 0043226, 55.34\%). In the biological process (BP), "cellular process" (GO: 0009987) enriched the most abundant unigenes (64.66\%). "metabolic process" (GO: 0008152) and "single-organism process" (GO: 0044699) enriched 57.42\% and 49.61\% unigenes, respectively. The distribution of GO terms in $\mathrm{KBG}$ indicated that the protein functions of the unigenes were from a diverse range of classes.

The result of KOG analysis showed that a total of 31,161 unigenes were annotated under 25 categories (Fig. S1). "General function prediction only" (R) held the most abundant unigenes (8038 unigenes, 25.8\%), followed by "Signal transduction mechanisms" (T) (3394 unigenes, $10.9 \%)$ and "Posttranslational modification, protein turnover, chaperones" (O) (2935 unigenes, 9.4\%). Some functional description about plant growth and development were also annotated more than 1000 unigenes, such as "Carbohydrate transport and metabolism" (G) (1603 unigenes, 5.1\%), "Secondary metabolites biosynthesis, transport and catabolism" (Q) (1294 unigenes, 4.15\%), "Amino acid transport and metabolism" (E) (1129 unigenes, 3.6\%), "Lipid transport and metabolism" (I) (1057 unigenes, $3.4 \%$ ) and so on.

Table 2. Annotation summary of all unigenes in public databases

\begin{tabular}{cccccc}
\hline Database & NR & SWISSPROT & KOG & KEGG & GO \\
\hline Annotation numbers & 55781 & 35425 & 30108 & 9201 & 29210 \\
Annotation ratio (\%) & 50.35 & 31.98 & 27.18 & 8.31 & 26.37 \\
\hline
\end{tabular}

Species_distribution

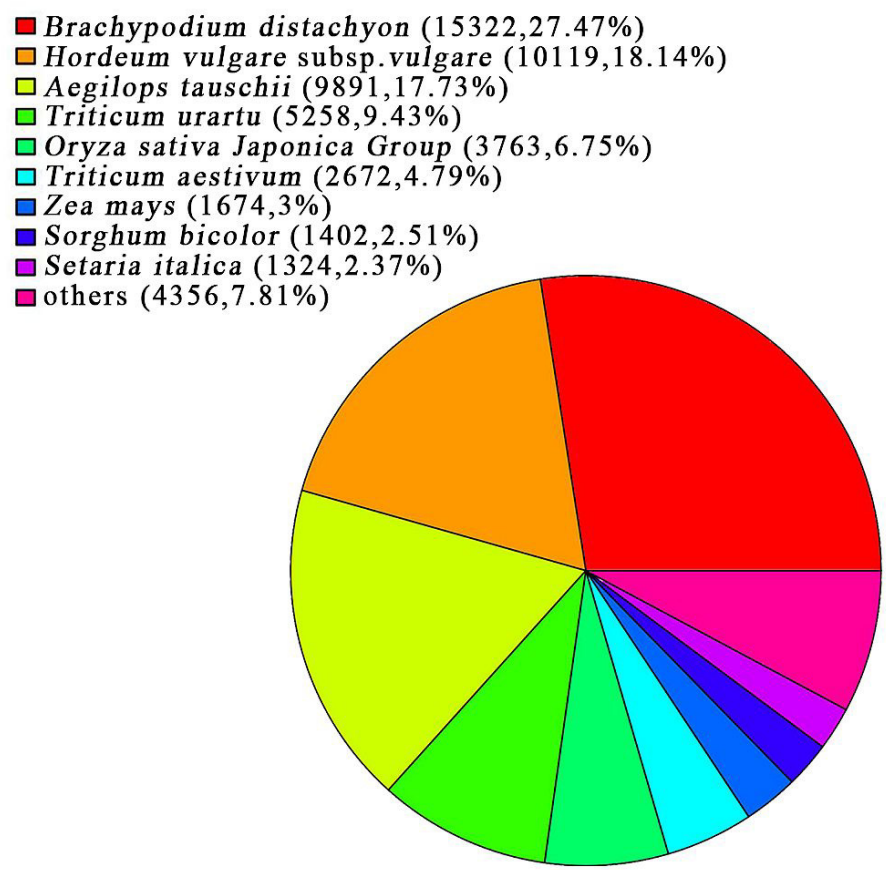

Fig. 1. Distribution species of the NR BLASTx top hits 
332

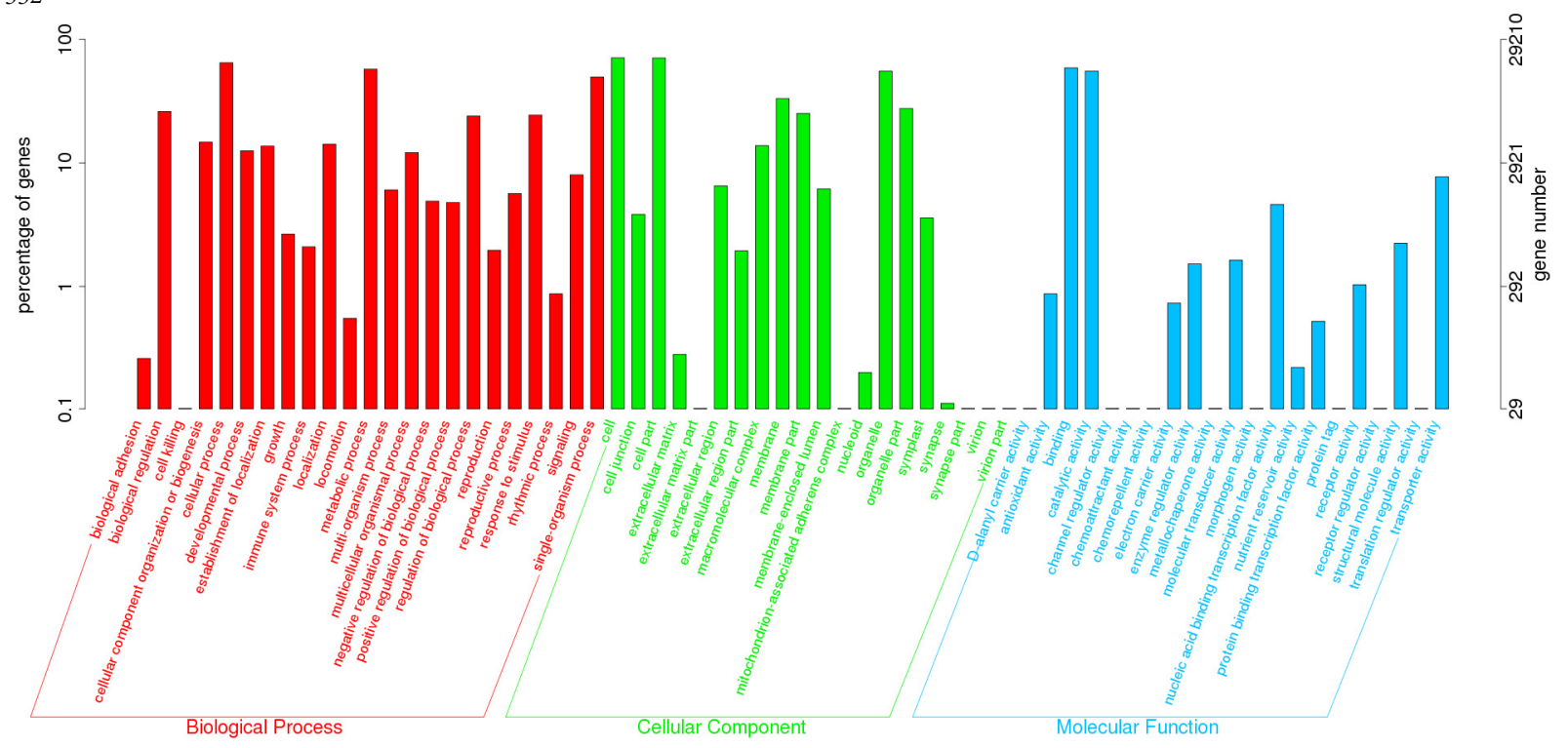

Fig. 2. Gene Ontology (GO) distributions for the Kentucky bluegrass transcriptome

\begin{abstract}
Analysis of DEGs between different heat stress time and genotypes

Based on the transcript abundance of all common unigenes from six samples, linkage hierarchical clustering analysis was conducted (Fig. 3). Depending on the diversity of the expression profiles from different genotypes and heat stress time, we identified the relationship of the six samples at transcriptional level. Cluster analysis showed that the expression profiles of the same stress time were grouped together and these of 03-0h and NL-0h samples had the closest relationship. The result suggested the transcriptional differences were mainly attributed to heat stress rather than genetic background. Heat map further showed that the expression profiles of $0 \mathrm{~h}$ and $24 \mathrm{~h}$ samples were more similar than these of $0 \mathrm{~h}$ and $2 \mathrm{~h}$ samples. It suggested that the expression profiles of short heat treatment had larger variety than long time treatment.
\end{abstract}

Further analysis of DEGs showed it was far more upregulated unigenes than down-regulated unigenes under heat stress (Fig. 4). After $2 \mathrm{~h}$ heat stress, more special upregulated differentially expressed unigenes (DEGs) and less down-regulated DEGs were detected in Ninglan (up: 2446; down: 379) than in KBG03 (up: 1514; down: 410). Meanwhile, there were 1364 up-regulated DEGs and 66 down-regulated DEGs were shared between them. At $24 \mathrm{~h}$, the expression of 1710 and 730 unigenes were increased and decreased specially in Ninglan, and 1361 up-regulated DEGs and 757 down-regulated DEGs were uniquely detected in KBG03. Another 696 and 341 DEGs were shared between the two genotypes with increased and decreased expression, respectively. Overall, more DEGs and up-regulated DEGs were identified at $2 \mathrm{~h}$ than $24 \mathrm{~h}$ in KBG. It was because the expression levels of some high-level expression unigenes (at $2 \mathrm{~h}$ ) were recovered to the normal levels (at 24h), such as heat shock protein (HSPs) unigenes and heat shock transcription factors (HSFs) unigenes. During the whole heat stress process, the down-regulated unigene numbers of the two genotypes were always similar, while more up-regulated unigenes were detected in Ninglan than KBG03 significantly. The Venn diagram (Fig. 4B) also showed 1433 and 1042 shared DEGs were detected in both materials at $2 \mathrm{~h}$ and $24 \mathrm{~h}$, respectively. The common upregulated unigenes at $2 \mathrm{~h}$ (1364) was nearly twice the number of $24 \mathrm{~h}$ (696), while the common down-regulated unigenes at $24 \mathrm{~h}(341)$ were much more than those at $2 \mathrm{~h}$ (66).

In the study, along with the heat stress proceeding, less DEGs and up-regulated DEGs were detected in both genotypes. However, more down-regulated DEGs were found at $24 \mathrm{~h}$. Similar result was reported in carnation (Dianthus caryophyllus L.) in response to heat stress, the number of DEGs reached a peak at $2 \mathrm{~h}$ and reduced at $12 \mathrm{~h}$. However, along with the stress increased, more decreased transcript abundance genes were identified only in heattolerant tall fescue materials ( $\mathrm{Hu}$ et al., 2014). The difference might be result from different resistance of the materials (and may also involve species, heat stress time, developmental stages, etc.).

\section{$G O$ enrichment of DEGs}

$\mathrm{GO}$ enrichment analysis of DEGs was conducted in different compared combinations, and the terms of BP ontology were analyzed in the subsequent analyses. As a whole, more enriched DEGs were detected in Ninglan than KBG03 and more DEGs enriched at $2 \mathrm{~h}$ than those at $24 \mathrm{~h}$. The result was similar with DEGs analysis in above.

Firstly, we compared the enriched GO terms from different stress time points (Table S2). In KBG03, there were six terms were consistent in two compared groups (03$0 \mathrm{~h}$ vs. 03-2h, 03-0h vs. 03-24h): "transcription, DNAtemplated", "protein folding", "response to heat", "response to high light intensity", "response to cyclopentenone" and "response to hydrogen peroxide". In Ninglan, four terms were common between two heat stress points (NL-0h vs. NL-2h, NL-0h vs. NL-24h): "protein folding", "response to heat", "defense response to bacterium, incompatible 
interaction" and "response to hydrogen peroxide". It was obviously that these unigenes relating to the functions of "protein folding", "response to heat" and "response to hydrogen peroxide" were regulated remarkably under heat stress in KBG.

The GO term of "response to heat" (GO: 0009048) included a total of 71 unigenes, 48 of them were shared in both materials. KBG03 included 54 (03-0h vs. 03-2h: 49; $03-0 h$ vs. $03-24 h: 28$ ) and Ninglan included 65 (NL-0h vs. NL-2h: 61; NL-0h vs. NL-24h: 37). The GO term of "response to hydrogen peroxide" (GO: 0042542) included 25 unigenes, in which 17 were common between the two genotypes. KBG03 had 19 (03-0h vs. 03-2h: 17; 03-0h vs. 03-24h: 12) and Ninglan had 23 (NL-0h vs. NL-2h: 20; NL-0h vs. NL-24h: 13). The GO term of "protein folding" (GO: 0006457) included 83 unigenes, and 55 were uniform in both materials. KBG03 included 65 (03-0h vs. 03-2h: 55; $03-0 h$ vs. $03-24 h: 34)$ and Ninglan included 73 (NL-0h vs. NL-2h: 66; NL-0h vs. NL-24h: 42). Most common unigenes involved in these GO terms had similar variation tendency. They were up-regulated after heat stress and the expression level was many times higher at $2 \mathrm{~h}$ than $24 \mathrm{~h}$.
Only two unigenes in GO: 0006457 appeared diverse trend of regulation: 1) comp148926_c0_seq2_1 had no significant expression change at $2 \overline{\mathrm{h}}$. Nevertheless, it was down-regulated significantly at $24 \mathrm{~h}(<5 \%$ in KBG03; $<8 \%$ in Ninglan). 2) In KBG03, the expression of comp146338_c0_seq1_1 raised 5764 folds and 465 folds at $2 \mathrm{~h}$ and $24 \mathrm{~h}$, respectively. But in Ninglan, it was downregulated to $2 \%$ at $2 \mathrm{~h}$, and up-regulated 75 times at $24 \mathrm{~h}$. Further analysis showed ten terms were the same in both materials at $2 \mathrm{~h}$ (03-0h vs. $03-2 \mathrm{~h}$; NL-0h vs. NL-2h) and nine were the same at $24 \mathrm{~h}$ (03-0h vs. $03-24 \mathrm{~h}$; NL-0h vs. NL-24h). It suggested these GO terms might be related to the basic thermal response mechanism in KBG.

Secondly, we analyzed the GO enrichment between the two genotypes at $0 \mathrm{~h}, 2 \mathrm{~h}$ and $24 \mathrm{~h}$ heat stress points (Table S3). Among the three compared groups (03-0h vs. NL-0h; 03-2h vs. NL-2h; 03-24h vs. NL-24h), there were twelve common GO terms: "DNA integration", "DNA recombination", "RNA-dependent DNA replication", "DNA biosynthetic process", "mitochondrial genome maintenance", "telomere maintenance", etc.

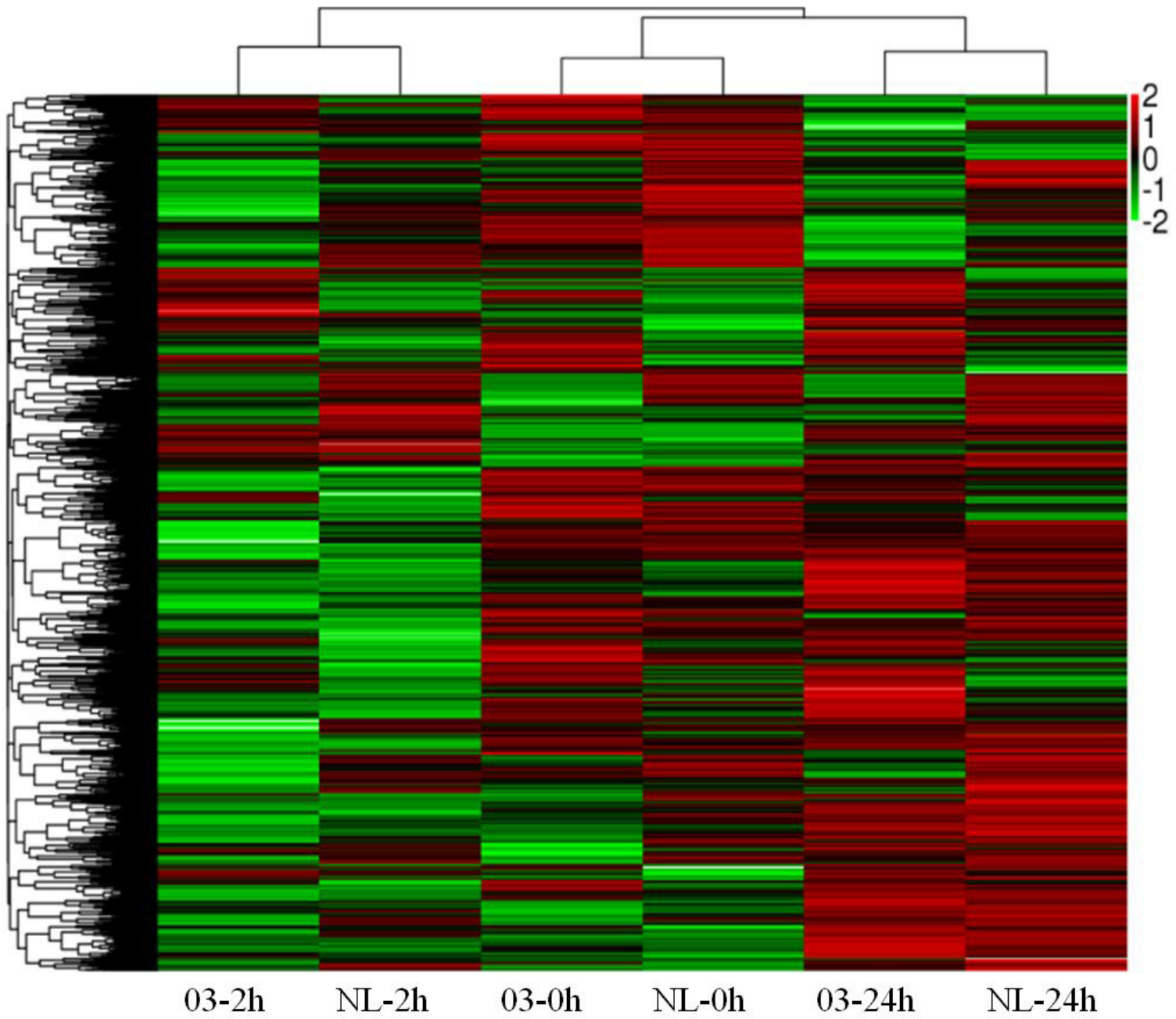

Fig. 3. Hierarchical clustering analysis of all common unigenes from six samples. Each column represents a different sample: 030h, 03-2h and 03-24h represent KBG03 under heat stress for $0 \mathrm{~h}, 2 \mathrm{~h}$ and $24 \mathrm{~h}$, respectively; NL-0h, NL-2h and NL-24h represent Ninglan under heat stress for $0 \mathrm{~h}, 2 \mathrm{~h}$ and $24 \mathrm{~h}$, respectively. Red: up-regulation; green: down-regulation; black: no change 


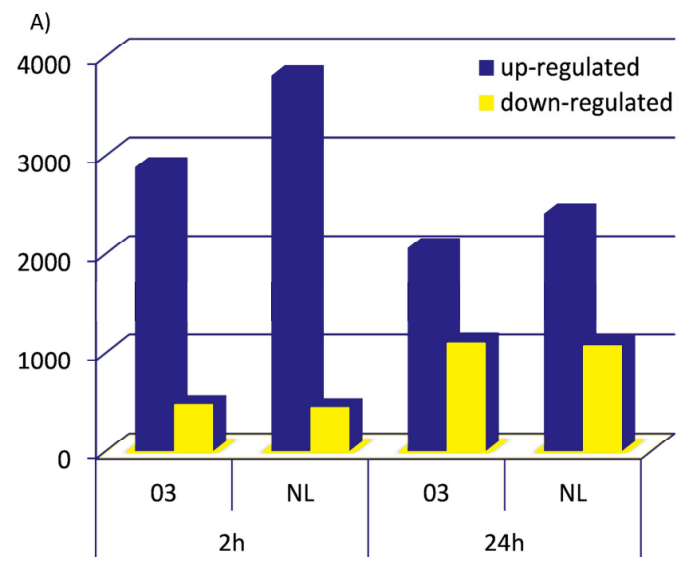

B)
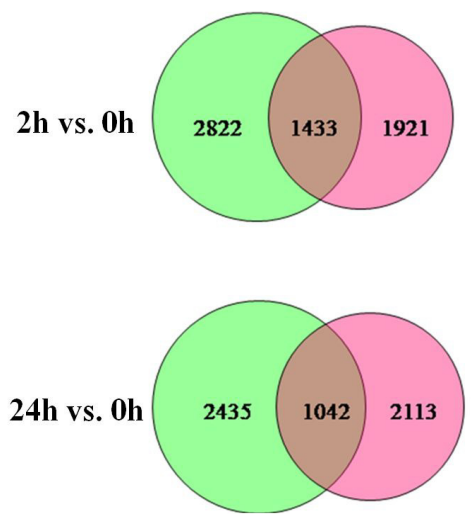

Down
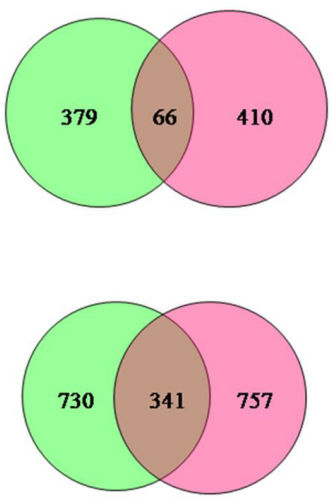

Up
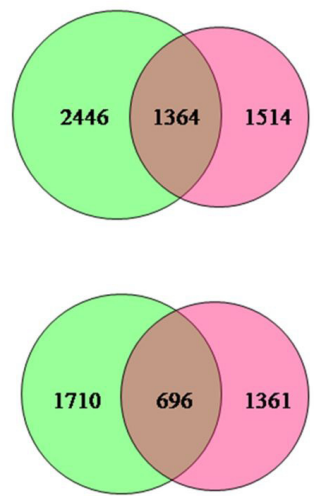

Fig. 4. Analysis of DEGs (fold change $>2, \mathrm{P}<0.05$ ) in $\mathrm{KBG}$ in response to heat stress. (A) Numbers of the significantly regulated unigenes of KBG03 and NingLan at $2 \mathrm{~h}$ and $24 \mathrm{~h}$ heat stress points. (B) Venn diagrams analysis of the DEGs at the two heat stress time points. Green circles denote Ninglan, Red circles denote KBG03

\section{HSPs in DEGs}

$H S P$ s play a central role in response to high temperature stress and thermotolerance acquisition in plants (Sun $e t$ al., 2014). HSPs would be synthesized upon multiple unfavorable environments, including heat, cold, salinity, UV-B light and anoxic stress (Driedonks et al., 2015). According to the annotation in NR and SwissProt databases, we analyzed the HSPs related unigenes in DEGs (Table 3). In KBG03, a total of 78 and 46 HSPs were separately found at $2 \mathrm{~h}$ and $24 \mathrm{~h}$. Among these, there were 11 $(2 \mathrm{~h})$ and 46 (24h) unigenes had no expression before heat stress. In Ninglan, 99 and 62 HSPs were separately detected at $2 \mathrm{~h}$ and $24 \mathrm{~h}$, in which $16(2 \mathrm{~h})$ and $8(24 \mathrm{~h})$ unigenes had no expression at $0 \mathrm{~h}$. Overall, more $H S P_{s}$ were induced at $2 \mathrm{~h}$ than $24 \mathrm{~h}$, and more $H S P s$ were detected in Ninglan than KBG03. The data demonstrated, in response to heat stress, more $H S P$ s showed significant differential expression in Ninglan than KBG03, and a large portion of them presented a strong response under short-term high temperature stress.

As molecular chaperones, HSPs could prevent protein aggregation and assist refolding of non-native protein by heat shock treatment (Xu et al., 2011). Plants could acquire thermotolerance by synthesizing a set of HSPs under high temperature (Howarth 1991). In the orchardgrass (Dactylis glomerata L.) (Huang et al., 2015), more unigenes were responded to heat stress in heat-resistant material. Also in tall fescue, they found the heat-tolerant sample had more DEGs relative to heat-sensitive one ( $\mathrm{Hu}$ et al., 2014). These response genes might participate in heat stress regulatory mechanisms in heat-tolerant grass. In this study, more DEGs and HSPs were abundant in the wild genotype material Ninglan than self-breeding line KBG03. So we speculated that Ninglan accumulated more heat response genes through a long-term acclimation process, and it might be more resistant to heat stress compared with KBG03. However, as mentioned in methods, Ninglan exhibited weaker summer stress tolerance than KBG03, so high temperature might not be the main environmental stress factor in the summer for these years in Shanghai and surrounding areas. Further single-factor experiments (including thermal stress, submergence stress, drought stress, UV-B stress, etc.) would be conducted to verify the tolerance of the two materials.

HSP are generally grouped into five classes named for their molecular masses (Vierling 1991): HSP100, HSP90, HSP70, HSP60, and small HSPs (sHSPs). A total of 93 HSPs DEGs (no HSP100 and HSP60 classes) were detected under heat stress treatments (03-0h vs. 03-2h; 03$0 \mathrm{~h}$ vs. $03-24 \mathrm{~h}$; NL-Oh vs. NL-2h; NL-0h vs. NL-24h) (Table S4). In our study, all the HSPs were grouped into three families (Howarth 1991):42 unigenes were low molecular weight (LMW: 15-30 k Da) HSPs; 33 unigenes 
were relating to $H S P 70(69-71 \mathrm{kDa}) ; 18$ unigenes were high molecular weight (HMW: $80-114 \mathrm{kDa}$ ) HSPs. All kinds of $H S P s$ were significantly up-regulated after subjecting to heat stress (Table S5), as reported in many plants ( $\mathrm{Hu}$ et al., 2014; Sun et al., 2014; Wan et al., 2015; Tian et al., 2009; Giorno et al., 2010; Li et al., 2011; Wang and Luthe, 2003). The numbers of LMW-HSPs DEGs were similar between $2 \mathrm{~h}$ and $24 \mathrm{~h}$ in both genotypes. However, HSP70 and HMW-HSPs DEGs were all obviously less detected at $24 \mathrm{~h}$ than 2h. Most HSPs exhibited the highest transcriptional level at $2 \mathrm{~h}$ heat stress time in carnation (Wan et al., 2015), which was consistent with our result.

In response to high temperature, thirteen HSPs DEGs were separately shared between the two genotypes at $2 \mathrm{~h}$ and $24 \mathrm{~h}$, which were more than those detected at optimum temperature (4) (Table 3). Further analyses of these HSPs (Table S6), two DEGs (comp131554_c0_seq5_1, comp63245_c0_seq1_2) were only detected at $0 \mathrm{~h}$, and they were separately related to HSP81 and HSP70. The other two heat shock cognate protein 70 (HSC70: comp192598_c0_seq21_2, comp199473_c0_seq1_2) DEGs were detected at both $0 \mathrm{~h}$ and $2 \mathrm{~h}$, and not found at 24h. Their expression was never detected in KBG03 all the time (0h, $2 \mathrm{~h}$ and $24 \mathrm{~h}$ ). It was reported that many HSP70 not only induced by stress, but also play a vital role in housekeeping activities under normal conditions. Moreover, some HSC70 (HSP70 homologs) are constitutively expressed in eukaryotic cytosol (Xu et al., 2011). We found HSPs DEGs between the two materials under normal temperature were mainly HSP70 and HSC70 indeed. Six HSPs had differential expression between the two genotypes at both heat stress time and five were unique at $2 \mathrm{~h}$ and $24 \mathrm{~h}$ respectively.

Under normal condition, the expression of CL26Contig1 was very low in both genotypes. After heat stress, its expression was sharply increased in KBG03 but not in Ninglan. Combined with the future resistance identification, these special HSPs DEGs could be focused for further study.

Transcription factors (TFs) in DEGs

Besides HSPs, TFs are also an abundant type of stressrelated genes (Nover et al., 2001). As the earliest group of responding genes to biotic and abiotic stresses, they play essential roles in signal transduction pathways (Zhang et al., 2005). Some families of TFs (e.g., bHLH, MYB, WRKY, ERF, HSF) are reported to be regulated under different stress responses in plants (Sun et al., 2014; Wan et al., 2015).
Among all types of TFs (Table 4), HSFs were the most (38) (03-0h vs. 03-2h: 24 ; 03-0h vs. 03-24h: 10 ; NL-0h vs. NL-2h: 31; NL-0h vs. NL-24h: 16) and they were all upregulated by high temperature treatment. HSFs were detected more largely at $2 \mathrm{~h}$ than those was detected at $24 \mathrm{~h}$ and more in Ninglan than in KBG03. In KBG03, 17 significantly up-regulated $H S F$ s were only detected at $2 \mathrm{~h}$, and 3 were just detected at $24 \mathrm{~h}$ (Table S4). In Ninglan, 17 differential expression $H S F$ s were only detected at $2 \mathrm{~h}$, and 2 were just found at $24 \mathrm{~h}$. In both genotypes, more HSFs DEGs were detected at $2 \mathrm{~h}$ than those at $24 \mathrm{~h}$. It indicated that $H S F$ s were early response genes to heat stress.

Base on the presence of conserved DNA-binding domains plus the nearby $\mathrm{HR}-\mathrm{A} / \mathrm{B}$ region amino acid structure, HSFs could be classified into three classes: A, B and C (Nover et al., 2001). In tomato (Solanum lycopersicum), as a critical gene, LeHsfAla appeared the master regulator function in heat stress response (Hahn et al., 2011), which formed a regulatory network with $H s f A 2$ and HsfBl (Mishra et al., 2002). In carnation, the expression levels of $H s f A 1, H s f A 2$ and $H s f B 1$ were also increased under heat stress (Wan et al., 2015). In Arabidopsis, expression of rice OsHsfA2e enhanced the tolerance to environmental stresses (Yokotani et al., 2008). In Chrysanthemum nankingense (Asteraceae), HsfA2 and $H s f A 3$ played an important role in the heat stress, but $H s f A l a$ and $H s f A 2$ were not detected (Sun et al., 2014). In this study, HsfA2, HsfA3, HsfA4, HsfA6, HsfA9, HsfB1, $H s f B 2, H s f C 1$ and $H s f C 2$ were all detected, except for HsfA1 (Table S7). Among them, HsfA2a, HsfA3, HsfA9, $H s f B 2 b$ and $H s f B 2 c$ were detected in all compared groups, but $H s f A 2 c$ and $H s f A 2 d$ were only detected at $2 \mathrm{~h}$ heat stress treatment. $H s f B 2 c$ and $H s f A 2 d$ hold the most number in all types of HSPs (seven and five were separately detected in Ninglan at $2 \mathrm{~h}$ ).

At the same heat stress time, more HSFs showed significant differential expression between the two genotypes at $24 \mathrm{~h}$ (9) than at $2 \mathrm{~h}(3)$, and no differentially expressed HSFs were detected at $0 \mathrm{~h}$ (Tables 3 and 4). Furthermore, more HSFs had higher expression in Ninglan (8) than those in KBG03 (3) (Table S6). Zhang et al. (2005) found prominently higher expression of TFs were detected in heat tolerant plants, especially in early heat stress condition. As the analysis in HSPs DEGs, we surmised Ninglan maybe has stronger resistance than KBG03 again. Significantly, most HSFs DEGs were only detected at one stress time, except for comp136900_c0_seq9_1. It had no expression in Ninglan all the time.

Table 3. Statistics of the DEGs related to HSPs and HSFs

\begin{tabular}{|c|c|c|c|c|c|c|}
\hline \multirow{2}{*}{ Compared group } & \multicolumn{3}{|c|}{$H S P s^{a}$} & \multicolumn{3}{|c|}{$H S F_{s}{ }^{a}$} \\
\hline & NR & Swiss-Prot & Total & NR & Swiss-Prot & Total \\
\hline 03-0h vs. $03-2 \mathrm{~h}$ & 38 & 69 & 78 & 12 & 24 & 24 \\
\hline 03-0h vs. $03-24 \mathrm{~h}$ & 18 & 43 & 46 & 5 & 10 & 10 \\
\hline NL-0h vs. NL-2h & 55 & 86 & 99 & 14 & 31 & 31 \\
\hline NL-0h vs. NL-24h & 27 & 55 & 62 & 8 & 16 & 16 \\
\hline 03-0h vs. NL-0h & 1 & 4 & 4 & 0 & 0 & 0 \\
\hline 03-2h vs. NL-2h & 4 & 13 & 13 & 0 & 3 & 3 \\
\hline 03-24h vs. NL-24h & 4 & 11 & 13 & 6 & 9 & 9 \\
\hline
\end{tabular}

${ }^{2}$ DEGs function was predicted by the annotation in NR and Swiss-Prot databases. Total: combined the unigenes annotated in both databases. 
Table 4. Statistics of several types of TFs in DEGs (by Swiss-Prot database)

\begin{tabular}{|c|c|c|c|c|c|c|c|}
\hline Type & $\begin{array}{c}\text { 03-0h vs. } \\
03-2 \mathrm{~h}\end{array}$ & $\begin{array}{c}\text { 03-0h vs. } \\
03-24 \mathrm{~h}\end{array}$ & $\begin{array}{c}\text { NL-0h vs. } \\
\text { NL-2h }\end{array}$ & $\begin{array}{c}\text { NL-0h vs. } \\
\text { NL-24h }\end{array}$ & $\begin{array}{c}\text { 03-0h vs. } \\
\text { NL-0h }\end{array}$ & $\begin{array}{l}\text { 03-2h vs. } \\
\text { NL-2h }\end{array}$ & $\begin{array}{c}03-24 h \text { vs } \\
\text { NL-24h }\end{array}$ \\
\hline MYB & 4 & 1 & 5 & 0 & 3 & 2 & 2 \\
\hline WRKY & 5 & 5 & 3 & 11 & 2 & 2 & 1 \\
\hline ERF & 7 & 7 & 7 & 5 & 2 & 2 & 3 \\
\hline HSF & 24 & 10 & 31 & 16 & 0 & 3 & 9 \\
\hline bHLH & 0 & 6 & 1 & 5 & 1 & 1 & 2 \\
\hline Total & 40 & 29 & 47 & 37 & 19 & 17 & 26 \\
\hline
\end{tabular}

qRT-PCR validation of the DEGs

To validate the expression profile data of the RNA-Seq results, ten common DEGs in all compared combinations (03-0h vs. $03-2 h ; 03-0 h$ vs. $03-24 h ;$ NL-0h vs. NL-2h; NL$0 \mathrm{~h}$ vs. NL-24h) were selected and examined by qRT-PCR (Fig. 5). According to the annotation in SwissProt database, the function of these unigenes were as follow: comp223146_c0_seq6_2 and comp148676_c0_seq4_1, encoding heat stress TFs; CL1 $177 \bar{C}$ Contigl, comp232094_c0_seq1_2 and comp136236_c2_seq4_1, encoding HSPs; comp214191_c4_seq 5 _2 and comp230124_0 seq1 2, encoding chaperone protein; comp150388_cl_seq13_1, encoding zinc finger protein; CL691Contig1, encoding floral homeotic protein APETALA 2; comp152630_c0_seq10_1, encoding peptidyl-prolyl cis-trans isomerase. qRT-PCR result showed almost all unigenes were up-regulated, except for CL691Contig1. In addition, at different heat stress treatment time, the change trends of these unigenes were consistent with RNA-Seq. So it indicated that the RNASeq data was accuracy and reliable.
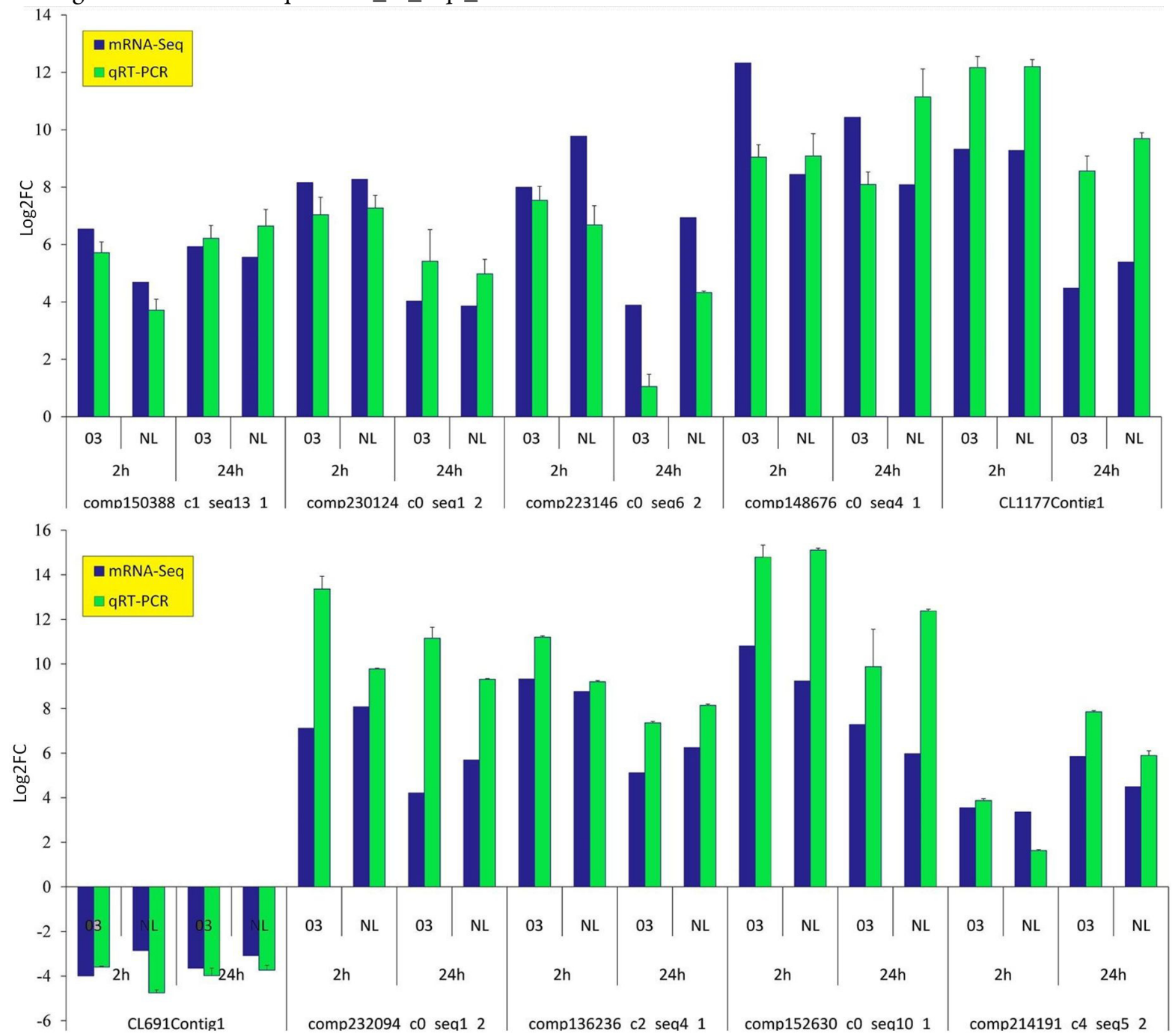

Fig. 5. qRT-PCR verification of ten differentially expressed unigenes 


\section{Conclusions}

This study represents the first RNA-seq analysis of KBG under heat stress treatments. A total of $81.42 \mathrm{~Gb}$ clean reads were de novo assembled into 110784 unigenes, and all unigenes were functionally annotated by NR, SwissProt, GO, KOG, and KEGG databases. Our data showed more DEGs, including HSPs and HSFs were detected under short heat treatment $(2 \mathrm{~h})$ than long time treatment $(24 \mathrm{~h})$, but less down-regulated DEGs were detected at $2 \mathrm{~h}$. Three core terms of BP ontology were found in both genotypes under different heat stress time. The DEGs shared in both genotypes might be related to the basic mechanism of thermal response in KBG and the unique DEGs in KBG03, or Ninglan, might be relevant to their difference in summer stress tolerance.

\section{Acknowledgements}

This work was funded by the National Natural Science Foundation of China (Grant \#: 31502002).

\section{References}

Ahn JH, Kim JS, Kim S, Soh HY, Shin H, Jang H, ... Huh JH (2015). De novo transcriptome analysis to identify anthocyanin biosynthesis genes responsible for tissue-specific pigmentation in zoysiagrass (Zoysia japonica Steud.).PLOSOne 10(4):e0124497.

Albertini E, Marconi G, Barcaccia G, Raggi L, Falcinelli M (2004). Isolation of candidate genes for apomixis in Poa pratensis L. Plant Molecular Biology 56(6):879-894.

Amaradasa BS, Amundsen K (2016). Transcriptome profiling of buffalograss challenged with the leaf spot pathogen Curvularia inaequalis. Frontiers in Plant Science 7:715.

An J, Shen XF, Ma QB, Yang CY, Liu SM, Chen Y (2014). Transcriptome profiling to discover putative genes associated with paraquat resistance in goosegrass (Eleusine indica L.). PLoSOne 9(6): e99940.

Bushman BS, Amundsen KL, Warnke SE, Robins JG, Johnson PG (2016). Transcriptome profiling of Kentucky bluegrass (Poa pratensis L.) accessions in response to salt stress. BMC Genomics 17:48.

Chen SY, Huang X, Yan XQ, Liang Y, Wang YZ, Li XF, ... Liu GS (2013). Transcriptome analysis in sheepgrass (Leymus chinensis): a dominant perennial grass of the Eurasian Steppe. PLoSOne 8(7):e67974.

Czaban A, Sharma S, Byrne SL, Spannagl M, Mayer KFX, Asp T (2015). Comparative transcriptome analysis within the Lolium/Festuca species complex reveals high sequence conservation. BMC Genomics 16:249.

Dinkins RD, Nagabhyru P, Graham MA, Boykin D, Schardl CL (2017). Transcriptome response of Lolium arundinaceum to its fungal endophyte Epichloe coenophiala. New Phytologist 213(1):324337.

Driedonks N, Xu JM, Peters JL, Park S, Rieu I (2015). Multi-level interactions between heat shock factors, heat shock proteins, and the redox system regulate acclimation to heat. Frontiers in Plant Science 6:999.

Farrell JD, Byrne S, Paina C, Asp T (2014). De novo assembly of the perennial ryegrass transcriptome using an RNA-Seq strategy. PLoS One 9(8):e103567.

Fox SE, Preece J, Kimbrel JA, Marchini GL, Sage A, Youens-Clark K, ... Jaiswal P (2013). Sequencing and de novo transcriptome assembly of Brachypodium sylvaticum (Poaceae). Applications in Plant Science 1(3):1200011.

Gan L, Di R, Chao YH, Han LB, Chen XW, Wu C, Yin SX (2016). De novo transcriptome analysis for Kentucky bluegrass bwarf mutants induced by space mutation. PLoSOne 11(5):e0151768.

Giorno F, Wolters-Arts M, Grillo S, Scharf KD, Vriezen WH, Mariani C (2010). Developmental and heat stress-regulated expression of HsfA2 and small heat shock proteins in tomato anthers. Journal of Experimental Botany 61(2):453-462.

Grabherr MG, Haas BJ, Yassour M, Levin JZ, Thompson DA, Amit I, ... Regev A (2011). Full-length transcriptome assembly from RNA-Seq data without a reference genome. Nature Biotechnology 29(7):644-652.

Hahn A, Bublak D, SchleiffE, ScharfKD (2011). Crosstalk between Hsp90 and $\mathrm{Hsp} 70$ chaperones and heat stress transcription factors in tomato. Plant Cell 23(2):741-755.

Howarth C (1991). Molecular responses of plants to an increased incidence of heat shock. Plant, Cell \&Environment 14(8):831-841.

Howarth C (2005). Genetic improvements of tolerance to high temperature. In: Ashraf M, Harris PJC (Eds). Abiotic stresses - plant resistance through breeding and molecular approaches. The Haworth Press, New York, pp 277-300.

Hu T, Sun XY, Zhang XZ, Nevo E, Fu JM (2014). An RNA sequencing transcriptome analysis of the high-temperature stressed tall fescue reveals novel insights into plant thermotolerance. BMC Genomics 15:1147.

Huang LK, Yan HD, Zhao XX, Zhang XQ, Wang J, Frazier T, ... Liu W (2015). Identifying differentially expressed genes under heat stress and developing molecular markers in orchardgrass (Dactylis glomerata L.) through transcriptome analysis. Molecular Ecology Resources 15(6):1497-1509.

Huang X, Yan HD, Zhang XQ, Zhang J, Frazier TP, Huang DJ, ... Yan YH (2016). De novo transcriptome analysis and molecular marker development of two Hemarthria species. Frontiers in Plant Science 7:496.

Huff DR (2003). Kentucky bluegrass. Turfgrass biology, genetics, and breeding.John Wiley \& Sons Hoboken, NJ,pp 27-38.

Kovi MR, Abdelhalim M, Kunapareddy A, Ergon A, Tronsmo AM, Brurberg MB, ... Rognli OA (2016). Global transcriptome changes in perennial ryegrass during early infection by pink snow mould. Scientific Reports 6:28702.

Li SF, Li F, Wang JW, Zhang W, Meng QW, Chen THH, ... Yang XH (2011). Glycinebetaine enhances the tolerance of tomato plants to high temperature during germination of seeds and growth of seedlings. Plant Cell and Environment 34(11):1931-1943.

Li YF, Wang YX, Tang YH, Kakani VG, Mahalingam R (2013). Transcriptome analysis of heat stress response in switchgrass (Panicum virgatum L.). BMC Plant Biology 13:153.

Meyer WA (1982). Breeding disease-resistant cool-season turfgrass cultivars for the United States. Plant Disease 66(4):341-344.

Mishra SK, TrippJ, Winkelhaus S, Tschiersch B, Theres K, Nover L, Scharf 
338

$\mathrm{KD}$ (2002). In the complex family of heat stress transcription factors, HSfAl has a unique role as master regulator of thermotolerance in tomato. Genes \&Development 16(12):1555-1567.

Ni Y, Guo N, Zhao QL, Guo YJ (2016). Identification of candidate genes involved in wax deposition in Poa pratensis by RNA-seq. BMC Genomics 17:314.

Nover L, Bharti K, Doring P, Mishra SK, Ganguli A, Scharf KD (2001). Arabidopsis and the heat stress transcription factor world: how many heat stress transcription factors do we need? Cell Stress Chaperon 6(3):177-189.

Orshinsky AM, HuJN, Opiyo SO, Reddyvari-Channarayappa V, Mitchell TK, Boehm MJ (2012). RNA-Seq analysis of the Sclerotinia homoeocarpa - creepingbentgrass pathosystem. PLoSOne 7(8):e41150.

Priest HD, Fox SE, Rowley ER, Murray JR, Michael TP, Mockler TC (2014). Analysis of global gene expression in Brachypodium distachyon reveals extensive network plasticity in response to abiotic stress. PLoS One9(1):e87499.

Puyang XH, An MY, Han LB, Zhang XZ (2015). Protective effect of spermidine on salt stress induced oxidative damage in two Kentucky bluegrass (Poa pratensis L.) cultivars. Ecotoxicology and Environmental Safety 117:96-106.

Sun J, Ren LP, Cheng Y, Gao JJ, Dong B, Chen SM, Chen FD, Jiang JF (2014). Identification of differentially expressed genes in Chrysanthemum nankingense (Asteraceae) under heat stress by RNA Seq. Gene 552(1):59-66.

Sung DY, Kaplan F, Lee KJ, Guy CL (2003). Acquired tolerance to temperature extremes. Trends in Plant Science 8(4):179-187.

Tian J, Belanger FC, Huang BR (2009). Identification of heat stressresponsive genes in heat-adapted thermal Agrostis scabra by suppression subtractive hybridization. Journal of Plant Physiology 166(6):588-601.

Toledo-Silva G, Cardoso-Silva CB, Jank L, Souza AP (2013). De novo transcriptome assembly for the tropical grass Panicum maximum Jacq. PLoSOne 8(7):e70781.

Trapnell C, Williams BA, Pertea G, Mortazavi A, Kwan G, van Baren MJ,... Pachter L (2010). Transcript assembly and quantification by RNA-Seq reveals unannotated transcripts and isoform switching during cell differentiation. Nature Biotechnology 28(5):511-515.

Vierling E (1991). The roles of heat shock proteins in plants. Annual review of plant biology 42(1):579-620.
Wachholtz M, Heng-Moss T, Twigg P, Baird L, Lu GQ, Amundsen K (2013). Transcriptome analysis of two buffalograss cultivars. BMC Genomics 14:613.

Wahid A, Gelani S, Ashraf M, Foolad MR(2007). Heat tolerance in plants: An overview. Environmental and Experimental Botany 61(3):199-223.

Wan XI, Zhou Q, Wang YY, Wang WE, Bao MZ, Zhang JW (2015). Identification of heat-responsive genes in carnation (Dianthus caryophyllus L.) by RNA-seq. Frontiers in Plant Science 6:519.

Wang DF, Luthe DS (2003). Heat sensitivity in a bentgrass variant. Failure to accumulate a chloroplast heat shock protein isoform implicated in heat tolerance. Plant Physiology 133(1):319-327.

Wei SJ, Du ZL, Gao F, Ke X, Li J, Liu JX, Zhou YJ (2015). Global transcriptome profiles of 'Meyer' Zoysiagrass in response to cold stress. PLoSOne 10(6):e0131153.

Weis E, BerryJA (1988). Plants and high temperature stress. Symposia of the Society for Experimental Biology 42:329-346.

Xu KJ, Sun FL, Chai GQ, Wang YF, ShiLL, Liu SD, Xi YJ (2015).Denovo assembly and transcriptome analysis of two contrary tillering mutants to learn the mechanisms of tillers outgrowth in switchgrass (Panicum virgatum L.). Frontiers in Plant Science 6:749.

Xu Y, Zhan C, Huang B (2011). Heat shock proteins in association with heat tolerance in grasses. International Journal of Proteomics 2011:529648.

Yang X, Yu XY, Li YF (2013). De novo assembly and characterization of the barnyardgrass (Echinochloa crus-galli) transcriptome using nextgeneration pyrosequencing. PLoSOne 8(7):e69168.

Yokotani N, Ichikawa T, Kondou Y, Matsui M, Hirochika H, Iwabuchi M, Oda K (2008). Expression of rice heat stress transcription factor OsHsfA2e enhances tolerance to environmental stresses in transgenic Arabidopsis.Planta 227(5):957-967.

Zhang L, Ren J, Li T, Wang A, Tan D (2016). De novo transcriptome sequencing of cold-treated Kentucky bluegrass (Poa pratensis) and analysis of the genes involved in cold tolerance. Journal of Horticulture 23:182.

Zhang Y, Mian MAR, Chekhovskiy K, So S, Kupfer D, Lai H, Roe BA (2005). Differential gene expression in Festuca under heat stress conditions. Journal of Experimental Botany 56(413):897-907. 